\title{
Teresa González Pérez. Templos del saber. Discursos políticos y utopías educativas. Madrid: Mercurio Editorial, 2015.
}

DOI: http://dx.doi.org/10.15648/hc.29.2016.10

Este libro ofrece un conjunto de trabajos de investigadores de Italia, Portugal, Brasil, Colombia, Chile, Estados Unidos y España que giran en torno a cómo se ha formado el magisterio, cómo han evolucionado las instituciones, las prácticas y las políticas educativas. Se abordan temas muy variados desde la formación del profesorado en determinadas épocas en Colombia, Brasil o Portugal, las ideas de Paulo Freire sobre la formación de los maestros, la incorporación de la asignatura Historia de la Educación en el curriculum para la formación docente en los Estados Unidos, la instalación de la orientación escolar a mediados del siglo XX en Chile o la evolución de las enseñanzas musicales en la educación obligatoria española.

En Le politiche educative in un'epoca di cambiamento sociale, Alessandro Bosi reflexiona sobre las aportaciones de la escuela a la sociedad. Tomando como punto de partida los discursos sobre políticas educativas de Lisboa 2000 el autor profundiza sobre el caso italiano desde los últimos años del siglo XX, recordando con ironía cómo muchos líderes políticos repitieron el mensaje de compromiso de Tony Blair cuando este afirmaba que quería concentrar en la educación los mejores recursos de su gobierno. La escuela es el lugar donde se unen el pasado y el futuro de un país, las necesidades sociales y los intereses de la producción, las generaciones y las poblaciones con diferentes valores, tradiciones y expectativas particulares, las personas con su propio sentido del tiempo y del espacio donde viven. Condicionada por las deficiencias del sistema público, la escuela es parte de la nueva lógica del Estado del Bienestar y el activo más importante para construir el futuro. 
En Ser modelo de virtud, de decoro y buenos modales para los alumnos. La formación de maestros en el Caribe colombiano durante el federalismo, Alarcón Meneses escribe sobre la creación de las Escuelas Normales en la segunda mitad del siglo XIX. La estatalización de la formación de maestros ocupó un lugar principal en la agenda educativa del liberalismo radical y con la reforma de 1870 la formación de maestros se convirtió en objetivo primordial. Para llevar a cabo esta reforma se diseñaron tres proyectos estrechamente relacionados: la creación de las escuelas normales en cada uno de los estados soberanos, la contratación de maestros alemanes para dar forma a lo que se llamó Misión Pedagógica Alemana y, por último, la publicación de periódicos y revistas de carácter instruccionista. En este texto se analizan algunos de los principales aspectos constitutivos de la cultura escolar propia de las escuelas de la región y, en concreto, la figura del maestro.

En Formação, história de vida e das práticas docentes de professoras do ensino primário no Sul do Brasil, 1930 a 1960: memórias de professoras, Ariclê Vechia y Antonio Gomes Ferreira recuperan las historias de las maestras que trabajaron desde 1930 hasta 1960 en las escuelas primarias públicas y privadas en el Estado de Paraná al sur de Brasil. Estas tres décadas, que coinciden con importantes cambios políticos en el país y con una época de fuerte influencia de la Escuela Nueva y de la Iglesia Católica, fue un momento de gran interés para la expansión de la escuela, pero con una educación desigual debido a las diferencias de clase, etnia, sexo y espacio geográfico.

Durante las primeras décadas del siglo XX, uno de los sueños de la sociedad brasileña, guiados por las ideas republicanas, era reformar la sociedad mediante la renovación del hombre; formar cívica y moralmente al pueblo brasileño con el objetivo de trasformar Brasil en una nación a la altura de las más progresistas del siglo. Para lograr estos objetivos era necesario ampliar la escuela primaria pública para todas las capas de la población, modificar los patrones de enseñanza y de las instituciones escolares para formar un contingente de trabajadores que atendiesen las necesidades de 250 la sociedad. La expansión y mejora de la formación del profesorado de educación primaria era indispensable para que el gobierno pudiese lograr su proyecto político-educativo. 
El texto está organizado en tres epígrafes en los que se mencionan las distintas misiones de estas maestras: inspirar más que enseñar, ser agentes de estandarización ideológica y cultural, y promover la transformación social. Las memorias de estas profesoras revelan que consideraban el magisterio como una misión y que estaban imbuidas de los deberes cívicos en el ejercicio de su profesión. Además, evidencian tener siempre un discurso magistral. Más que hablar de su docencia, destacan con orgullo su participación en la inspección de la educación, con miras al mantenimiento de la calidad de la enseñanza y la homogeneización ideológica y cultural y el hecho de que se tomaran este trabajo como una misión: la de transmitir a los niños la cultura y los hábitos tanto de la vida urbana, como de higiene, las normas de conducta y el amor por la patria.

En Formare un docente produttore e organizzatore per la scuola dell'autonomia, Anita Gramigna reconstruye el perfil del maestro italiano entre las décadas de 1970 a 1990 del siglo pasado a partir de la entrada en vigor de la ley sobre la autonomía escolar (Ley 23/12/1998, n. 448), especialmente a la luz de un programa de capacitación que se convirtió en el escenario de un intenso debate político e ideológico en torno al paradigma de la autonomía y la productividad. Al examinar el desarrollo de modelos de formación de profesionales de la enseñanza, esta autora reflexiona sobre la calidad educativa de la escuela, de acuerdo con la eficiencia y la eficacia de la gestión, al tiempo que compara la situación de la educación italiana con la española.

El siguiente trabajo se titula $A$ formação de professores na perspectiva de Paulo Freire: Desafios de uma pedagogia interdisciplinar. En el mismo Jaime Zitkoski examina la propuesta a favor de la pedagogía interdisciplinaria de uno de los pedagogos más significativos del siglo XX, Paulo Freire. Este educador propone una pedagogía innovadora para superar el modelo tradicional que reproduce la lógica de la fragmentación y la transmisión pasiva del conocimiento. Desde una perspectiva interdisciplinar, el objetivo central de la pedagogía de Freire es la lucha para transformar la realidad social y construir así un mundo más justo y fraterno.

En esta contribución se destacan las principales razones que Freire apunta para desarrollar una Pedagogía Interdisciplinar, que converge en la defensa 
de una educación humanista libertaria frente a la cultura de la opresión y de las prácticas sociales que reproducen la lógica de la dominación social. Jaime Zitkoski explica los principales argumentos de la propuesta de Freire en defensa de la interdisciplinariedad en la educación, la producción de conocimiento y la formación de los docentes. La coherencia de Freire desafía la construcción de alternativas en todos los niveles educativos y la praxis política para hacer frente a las diversas formas de opresión y de violencia contra las personas y la naturaleza. En este trabajo se destacan tres aspectos fundamentales de la propuesta de Freire que apuntan a la necesidad de construir una educación interdisciplinaria comprometida con la humanización del mundo: la ruptura con los modelos tradicionales de la ciencia y la educación, los retos de una pedagogía interdisciplinaria en la lectura del mundo contemporáneo, y la práctica del diálogo y la formación de maestros con vistas a la lucha política.

En el trabajo Formação de professores em Portugal (1970-1990) Maria João Mogarro relata los antecedentes y las circunstancias de la formación del magisterio en una etapa llena de transformaciones políticas y sociales. En un contexto que abarca desde la dictadura, la Revolución de los Claveles y el establecimiento de la democracia, esta autora hace un recorrido por las políticas educativas desde 1970, la reforma de Veiga Simão aprobada en 1973 hasta el restablecimiento de la democracia y la eliminación de las estructuras heredadas de la dictadura de Salazar, en vigor hasta abril de 1974. El trabajo incluye diversos apartados sobre la evolución de los planes de estudio y la organización de los cursos, las modalidades de evaluación y los exámenes, además de un epígrafe sobre la precaria situación institucional en la que se encontraban los profesores de estas escuelas de formación.

Durante su existencia las escuelas de Magisterio fueron consideradas instrumentos privilegiados de la política educativa de Salazar. Posteriormente, fueron también uno de los símbolos más fuertes de la educación socialista en su proceso revolucionario. Su final fue dictado por las organizaciones extranjeras ya que las nuevas instituciones traerían más financiación internacional para instalaciones, equipo y personal de formación de las escuelas. El proceso de la extinción gradual de estas escuelas de Magisterio primario en la década de los 80 marcó el fin de un ciclo en la formación de profesores y la cultura pedagógica portuguesa. 
En Innovación tecnocrática y nuevos saberes académicos en la formación docente, González Pérez analiza las transformaciones de los estudios de magisterio en España desde la década de los años 60 del pasado siglo XX hasta la actualidad. En el periodo tardo-franquista el gobierno español comienza a interesarse por mejorar el nivel cultural y por extender la escolarización como un medio de alcanzar el progreso económico. Con el Plan de 1971 los maestros pasan a denominarse profesores de educación general básica y las escuelas de Magisterio se integran en las universidades. Con rango de diplomatura universitaria, los estudios para la formación de los futuros maestros constarán de un solo ciclo que durará tres años, incluyendo un periodo de tres meses de prácticas de enseñanza. Se eleva la edad y la formación académica exigida para matricularse, siendo necesario obtener el título de COU (Curso de Orientación Universitaria). Se combina la formación de maestro generalista, capacitado para impartir enseñanzas en la primera etapa de la EGB, y la de maestros especialistas, para cada una de las áreas establecidas en la segunda etapa (Ciencias Sociales, Lengua, Ciencias Naturales y Matemáticas, principalmente). Este proceso de innovación tecnocrática aplicada a las culturas escolares bajo el control del Estado pretendía modernizar el sistema educativo sin cuestionar las bases ideológicas y políticas del franquismo.

La implantación de la Ley General de Educación (1970) supuso una profunda reforma de todo el sistema educativo español, constituyó la base para las reformas educativas posteriores, y sobre ella se asentó la Ley de Ordenación General del Sistema Educativo (1990) y el nuevo diseño curricular para el magisterio. En este capítulo González Pérez aborda el avance que significó para la educación española todas estas transformaciones que marcaron el tránsito educativo de la dictadura a la democracia.

En el capítulo Formação de professores e fortalecimento da escola pública no Brasil (1970-1990), Libania Xavier y Sonia de Castro Lopes dan una visión general de las políticas y experiencias de formación del profesorado en el contexto brasileño de las décadas de 1970-1990. Comenzando este recorrido histórico en el último tercio del siglo XX, estas autoras escriben sobre la evolución del magisterio en Brasil y su paralelismo con la enseñanza primaria. En la historia de la educación brasileña, la formación del profesora- 
do siempre ha ocupado un lugar destacado, ya sea en los discursos de los políticos e intelectuales más sobresalientes del siglo XIX y el paso a las primeras décadas del siglo XX, o en el periodo comprendido entre los años 1930 a la década de 1960 del siglo XX. Desde la década de 1980, la formación inicial y continua de los docentes se benefició de las innovaciones pedagógicas que marcaron el periodo de democratización del país con la institucionalización de algunos de sus principios básicos en las directrices legales establecidas en la Ley de Educación Superior, promulgada en 1996.

En su trabajo Scholars of Distinction and the Development of Educational History in the United States, Karl Lorenz estudia la incorporación de la asignatura Historia de la Educación en el curriculum para la formación docente desde mediados de siglo XIX. Este trabajo repasa brevemente las aportaciones de un grupo de eruditos que influyeron en el desarrollo del campo de la Historia de la Educación en los Estados Unidos. Los efectos de este trabajo sobre la teoría y la práctica educativa varían al igual que la influencia que ejercieron en diferentes momentos del pasado de ese país. Si bien la literatura especializada normalmente cita estas personalidades, un sinnúmero de otros académicos e instituciones también han dejado su huella en el campo como representantes de una comunidad más grande que ha estado activa desde mediados del siglo XIX, tanto los estudiosos reconocidos como los que no han establecido la historia de la educación como un importante campo de estudio y de investigación, y una parte integral de los programas de preparación para los educadores.

En Cambios en el desempeño profesional de la inspección educativa en los últimos cincuenta años, Salmerón Nieto, González García y Beas Miranda reflexionan sobre la Inspección educativa en España desde sus inicios en marzo de 1849 y presentan las transformaciones que ha experimentado este organismo en España en el último medio siglo a tenor de los avances sociales, políticos y educativos. La inspección educativa ha ido evolucionando a lo largo de su existencia acorde con las necesidades del sistema educativo y en ocasiones siguiendo los intereses de los diferentes grupos políticos en el poder. Los autores estudian las causas de los cambios realizados en la asignación de desempeños profesionales, estudian las funciones asignadas a esta inspección y las consecuencias de los cambios en sus atribuciones 
y competencias, y analizan el organigrama de este cuerpo de la administración.

Tras profundizar en los principales aspectos en los que se localizan cambios en la asignación de atribuciones y competencias desde 1970, los autores focalizan su trabajo en el desempeño profesional del equipo técnico de Inspección de Granada entre 1970 y 1977 con el objetivo de conocer el discurrir concreto de un servicio provincial, apreciando las posibles similitudes con lo visto con anterioridad sobre el conjunto del territorio español.

En el capítulo ¿Técnicos de la política y del alma? Un episodio en las discusiones sobre politicas de educación secundaria en Chile y la instalación de la orientación escolar a mediados del siglo XX, Pablo Toro Blanco examina el Plan de Renovación Gradual de la Enseñanza Secundaria (1945) tanto como un episodio de la política educacional, considerando los componentes domésticos del conflicto, como un síntoma de la internacionalización de las políticas de educación. A través del análisis de la reforma educativa en Chile de mediados de la década de 1940, el autor aborda la contraposición existente en la discusión local sobre políticas educativas entre visiones técnicas y sapienciales o de sentido común. Toro Blanco examina los aspectos de la integración de un nuevo programa curricular, la orientación educacional en la enseñanza secundaria chilena, una especialidad académica vinculada a la psicopedagogía que perseguía esencialmente la diversificación en sus áreas de intervención educativa con el alumnado.

Este trabajo se inscribe en el área de estudio de la historia de las políticas educativas y se complementa con un enfoque panorámico que explora la posibilidad de conectar con la historia de las emociones en la enseñanza secundaria. Para el investigador, la historia de las emociones ha traído a la historiografía nuevos y complejos desafíos, entre los que se incluye el carácter elusivo del objeto de estudio definido desde campos distantes como la biología y la antropología. En este sentido, en sus reflexiones finales Pablo Andrés Toro Blanco recuerda cómo pocos años después de establecerse los dispositivos curriculares de orientación educacional, tras los impulsos reformistas iniciados por el Plan de Renovación Gradual de 
la Enseñanza Secundaria, un analista profesional de psicología juvenil se lamentaba de que ciertas formas tradicionales de expresión de la identidad de los jóvenes, incluyendo los diarios de vida, estuvieran desapareciendo. Para el autor de este capítulo, esos serían un material muy enriquecedor tanto desde un punto de vista histórico como para la historia de las emociones.

Finalmente, en La evolución de las enseñanzas musicales en la educación obligatoria. De la LGE a la LOGSE, Juan Ramón Coello Martín hace un repaso a la inclusión de la asignatura de música en la enseñanza obligatoria en España. Aunque en la Ley sobre Educación Primaria de 1945 aparece la materia "Música y Canto", esta se incluye en el apartado de materias complementarias, de nivel secundario, y cuyos contenidos no están reglados por el Ministerio de Educación, Cultura y Deporte sino por diversos organismos competentes. Posteriormente, en las Orientaciones Pedagógicas de 1970-71 y de 1976 que desarrollan la Ley General de Educación de 1970 aparece el Área de Expresión Dinámica para la primera etapa de la Educación General Básica en la que se incorpora la asignatura de Música; si bien, según este investigador, en la praxis el Área de Expresión Dinámica quedó reducida a Expresión Corporal. A partir de 1990, con la entrada en vigor de la Ley Orgánica de Ordenación General del Sistema Educativo (LOGSE, 1990) se produjo la implantación curricular de la Música en la Enseñanza Obligatoria; en Educación Infantil, dentro de Comunicación y Representación; en Educación Primaria integrada en el ámbito de Expresión Artística, y en la Educación Secundaria dentro del Área de Música.

En este trabajo Coello Martín incluye los objetivos generales, los contenidos y los objetivos de las actividades de los distintos bloques temáticos que debían impartirse en los Ciclo Inicial, Medio y Superior de la Ley General de Educación, para posteriormente analizar la presencia de esta asignatura en la LOGSE, mencionando los objetivos de dicha asignatura tanto para la Educación Primaria como para la Educación Secundaria Obligatoria.

En definitiva, Templos del Saber. Discursos políticos y utopias educativas ofrece un conjunto valioso y muy variado de aportaciones sobre la historia del Magisterio en Italia, Portugal, Brasil, Colombia, Chile y España. La histo- 
riografía educativa se ha ido enriqueciendo con diversos estudios sobre las políticas públicas, los centros y niveles educativos realizados en diversas partes del mundo; estudios que han servido de base para preparar trabajos de síntesis con nuevos métodos y enfoques historiográficos. En el trabajo que nos ocupa, estas contribuciones ofrecen elementos para la comprensión global de las políticas educativas y de los proyectos educativos normalistas, y nos permiten comprender en qué coyunturas se transformó la educación y qué estrategias políticas se emplearon. La diversidad de estas aportaciones tanto en lo que respecta a enfoques como a la temática enriquecen la visión historiográfica de conjunto sobre un aspecto tan importante en la historia de la educación como es la formación de los maestros de enseñanza primaria.

María Jesús Vera Cazorla

Doctora en Filología Moderna Universidad de Las Palmas de Gran Canaria mariajesus.vera@ulpgc.es 